\title{
Design and Control of a Low-Cost Robotic Hand Using the Robot Operating System *
}

\author{
Walter Fetter Lages* Gabriel Figueiredo Schmitz* \\ Renato Ventura Bayan Henriques* \\ * Departamento de Sistemas Elétricos de Automação e Energia, \\ Universidade Federal do Rio Grande do Sul, (e-mail: \\ fetter@ece.ufrgs.br, gschmitz@ece.ufrgs.br, rventura@ece.ufrgs.br).
}

\begin{abstract}
This paper presents the design and control of a low-cost robotic arm using the Robot Operating System. Contrariwise to many works in ROS, the inertia parameters of the hand are realistic and the controller is tuned based on the equivalent transfer function of the joint, instead of by experimentation as suggested in many tutorials on using controllers in ROS. The results show that by properly tuning the controller and the Gazebo simulation parameters, the divergence problems that usually arise in the simulator when the inertia parameters are not realistic can be avoided.
\end{abstract}

Keywords: Humanoid Hand; Robotic Hand; Hand Design; Hand Control; Robot Operating System.

\section{INTRODUCTION}

In order to manipulate small objects, the hand executes a range of motions known as precision prehension (Napier, 1980). One of those prehensions, the bidigital between the thumb and index fingers is the most usual prehension, responsible for about $20 \%$ of the manipulations in daily activities (Magee, 1997).

The knowledge of the human hand is required for the design of robotic devices replicating its functions. Some works (Becker et al., 1986; Bucholz and Armstrong, 1992) disregard some anatomic details, while others are complex regarding the anatomy (Taylor and Schwartz, 1955), physiology (Kapandji, 2009) or kinesiology (Brook et al., 1995; An et al., 1979). By considering the articular, muscular and nervous systems of the human hand it is possible to develop a robotic hand and try to reproduce the human motion through actuating systems and proper control systems (Cutkosky, 1989; Hasser and Cutkosky, 2002).

This work proposes the development of a low cost hand which is able to perform prehensions of objects in many geometric forms. A control system based on the open source Robot Operating System (ROS) is developed and the performance on some typical prehensions is presented.

In the last decade, the Robot Operating System (ROS) has emerged as the main framework for research in Robotics (Cousins, 2010). It is available open source and has a strong community support.

The standard way to describe a robot to be used in ROS is through the Unified Robot Description Format (URDF), which is an XML extension for describing robots, including its kinematics and dynamics properties, visual

\footnotetext{
^ This study was financed in part by the Coordenação de Aperfeiçoamento de Pessoal de Nível Superior - Brasil (CAPES) - Finance Code 001.
}

representation and collision model. Some CAD programs include URDF exporters.

The organization of the this work is as follows: in the next section the proposed low cost hand is presented and its kinematic model using the modified Denavit-Hartenberg convention is presented. The actuators used to move the fingers are then modeled, enabling the development of a description of the hand in the Unified Robot Modeling Format (URDF). A simplified mathematical model, based on an equivalent inertia and a equivalent viscous friction is developed for each finger and used for the design of the controller gains. Then, the hand is simulated in Gazebo using the controllers implemented in ROS and its performance in reference tracking is analyzed. Finally, the conclusions and some ideas for the sequence of the work are presented.

\section{THE PROPOSED LOW-COST HAND}

In order to reduce the actuation needs and enable its implementation in dimensions similar to a human hand, the proposed hand has a reduced number of degrees of freedom (DoF) when compared to a human hand. It called Miitzhand and has 15 joints but only 6 DoFs. The three joints of each finger are commanded by a single cable, with exception of the thumb which has two DoFs.

Some characteristics of the mechanical design are:

- Fingers with smooth and round edges, to reduce the mobility restrictions of the joints;

- Guide channels for the actuation cables of each link, as seen in Figure 1, to improve the stiffness in actuation;

- Two actuating cables in each joint, one for tension motion and other for the relax motion;

- Physical limits to avoid fingers moving beyond relax position. 


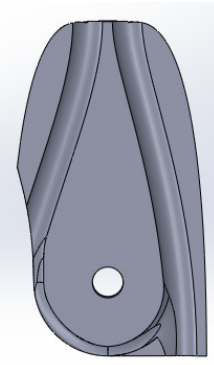

(a) Distal.

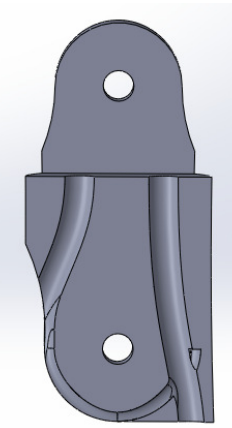

(b) Medium.

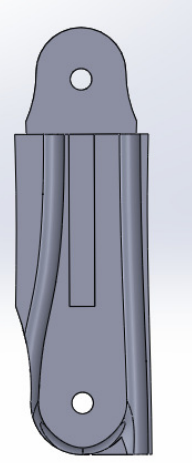

(c) Proximal.
Figure 1. 3D model of the phalanges.

Details of the cabling assembly for the index finger can be seen in Figure 2.

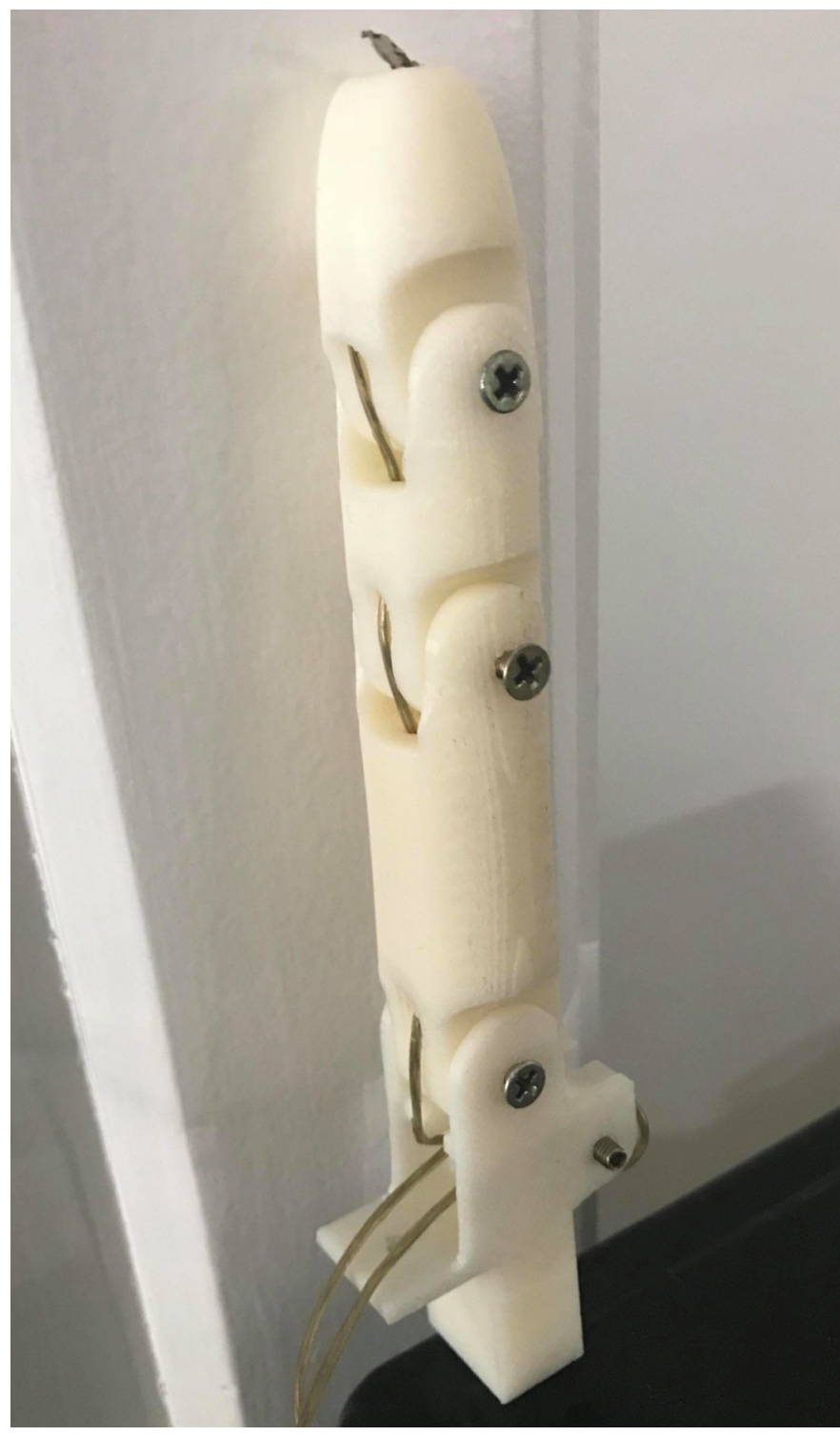

Figure 2. Detail on cabling assembly for the index finger.

Here, the hand CAD model was developed with the SolidWorks CAD, which has an exporter for URDF (ROS.org,
2013). However, the URDF description exported directly from the CAD program is not readily usable in ROS. Some manual tweaking is necessary to obtain a working URDF description. Furthermore, some care in necessary in the definitions of coordinate frames while exporting the CAD model to URDF. The conventions for coordinate frames used by SolidWorks are not the same used by URDF.

Besides that, for the Miitzhand it was chosen to use Modified Denavit-Hartenberg conventions (Craig, 1989) for the assignment of coordinate frames.

\subsection{Kinematic model}

The classical method to develop a kinematic model for articulated robots is to use the Denavit-Hartenberg convention (Fu et al., 1987). It is not necessary at all to use it to build an URDF model of the robot, but its use simplifies the structure of the homogeneous transforms between adjacent frames, making it easier to algebraically manipulate some expressions, as those required to develop the dynamic model using the Lagrange-Euler or NewtonEuler formalisms (Fu et al., 1987). However, the standard Denavit-Hartenberg convention is not directly compatible with URDF description. Standard Denavit-Hartenberg convention requires the $i$ joint to move (either rotate or translate) on the $Z_{i-1}$ axis, while in URDF the $i$ joint should move on an (arbitrary defined) axis in the $i$ frame . Of course, this incompatibility could be handled by creating intermediate frames, but that could potentially double the number of required frames, making the kinematic tree much larger.

A better alternative is to use the modified DenavitHartenberg convention (Craig, 1989), which requires the $i$ joint to move on the $Z_{i}$ axis. Nonetheless, this convention also requires the $X_{i}$ axis to intercept the $Z_{i+1}$ axis. For the frames associated with the fingers, this is not a problem as each frame has just one subsequent frame in the kinematic chain. However, for the frame associated with the base of the hand, there is a problem, as this frame has five subsequent frames, one for each finger. And, of course, it is not possible to choose a single $X$ axis for the base frame that intercepts the $Z$ axis of each finger. This happens because the modified Denavit-Hartenberg convention was developed to describe kinematic chains and not kinematic trees, hence, it does not support bifurcations. The hand is a kinematic tree, where each finger forms a kinematic chain starting at the hand base.

Note that the standard Denavit-Hartenberg convention does not have this problem as it requires the $Z_{i}$ axis to intercept the $X_{i+1}$ axis, which can be chosen in a way to ensure the existence of the required interceptions, as each subsequent coordinate system has its own $X$ axis. However, as already explained, the standard DenavitHartenberg convention is not directly compatible with URDF.

Again, the solution is to create intermediate frames, but now, it is enough to create just one intermediate frame for each finger such that their $X$ axis intercept the $Z$ axis of the first frame of each finger and their $Z$ axis are intercepted by the $X$ axis of the hand base frame. This can be done by defining those intermediate frames with 
its origin coincident with the first frame of each finger, the $X$ axis coincident with the $X$ axis of the first frame of each finger, but with the $Z$ axis rotated (around $X$ ) to in order to intercept the $X$ axis of the hand base system. This way, the plane defined by the $Z$ axis of the first coordinate system and the $Z$ axis of the respective intermediate frame is normal to the $X$ axis of the intermediate frame.

Those intermediate frames are created in URDF by defining a link without mass, visual or collision properties and a joint between the hand base link and the first link of each finger.

Figure 3 shows the assigned frames in RViz. The modified Denavit-Hartenberg parameters are shown in Table 1. Note that for each finger there is just one joint variable $\left(-180^{\circ} \leq \theta_{i} \leq-90^{\circ}\right)$, for the first joint of the finger. The other joints of the finger move coordinated with the first joint and have a value of $\theta_{i}+90^{\circ}$.

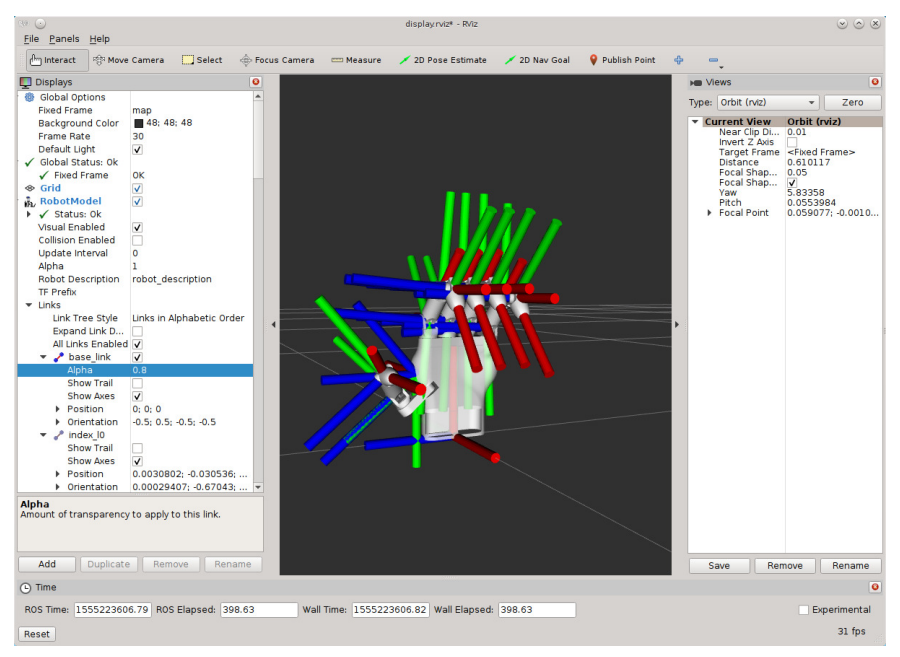

Figure 3. Hand frames.

\subsection{Actuator model}

Each fingers is actuated by a single gear-head D.C motor, which nominal data, considering the gearbox, is shown in table 2. Some other parameters of the actuator were measured in experiments and are detailed in table 3 .

Based on values in tables 2 and 3, other characteristics of the actuator can be computed. The reduction $n$ is given by:

$$
n=\frac{d_{m}}{d_{j}}
$$

which leads to a maximum velocity and nominal torque on the joints given by:

$$
\begin{aligned}
\omega_{\max } & =n \omega_{0} \\
\tau_{j N} & =\frac{\tau_{n}}{n}
\end{aligned}
$$

The armature constant can be computed by:

$$
K_{a}=V_{N}-i_{0} R_{a} \omega_{0}
$$

Table 4 shows the computed characteristics of the motor. Note that for a permanent magnet D.C. motor, and considering the S.I. units, the armature constant $K_{a}$ is

\begin{tabular}{|c|c|c|c|c|}
\hline$\overline{\text { link }}$ & $\overline{\theta_{i}\left(^{\circ}\right)}$ & $a_{i}(\mathrm{~mm})$ & $\overline{d_{i}(\mathrm{~mm})}$ & $\alpha_{i}\left(^{\circ}\right)$ \\
\hline origin_link & - & 0 & - & 0 \\
\hline \multicolumn{5}{|l|}{ Little chain } \\
\hline base_link & 90 & 119.77 & 0 & -2 \\
\hline little_10 & 90 & -1.5817 & -40.7 & 5 \\
\hline little_11 & $-180 \leq \theta_{l} \leq-90$ & 47 & 0 & 0 \\
\hline little_12 & $\theta_{l}+90$ & 26.7 & 0 & 0 \\
\hline little_13 & $\theta_{l}+90$ & - & 0 & - \\
\hline \multicolumn{5}{|l|}{ Ring chain } \\
\hline ring_10 & 90 & 3 & $-17,716$ & 2 \\
\hline ring_11 & $-180 \leq \theta_{r} \leq-90$ & 47 & 0 & 0 \\
\hline ring_12 & $\theta_{r}+90$ & 26.7 & 0 & 0 \\
\hline ring_13 & $\theta_{r}+90$ & - & 0 & - \\
\hline \multicolumn{5}{|l|}{ Middle chain } \\
\hline middle_10 & 90 & 3.4207 & 6.8043 & 0 \\
\hline middle_l1 & $-180 \leq \theta_{m} \leq-90$ & 47 & 0 & 0 \\
\hline middle_12 & $\overline{\theta_{m}}+90$ & 26.7 & 0 & 0 \\
\hline middle_13 & $\theta_{m}+90$ & - & 0 & - \\
\hline \multicolumn{5}{|l|}{ Index chain } \\
\hline base_link & 90 & 128.28 & 0 & 5.8 \\
\hline index_10 & 90 & 3.0963 & 30.691 & -1 \\
\hline index_11 & $-180 \leq \theta_{i} \leq-90$ & 47 & 0 & 0 \\
\hline index_12 & $\theta_{i}+90$ & 26.7 & 0 & 0 \\
\hline index_13 & $\theta_{i}+90$ & - & 0 & - \\
\hline \multicolumn{5}{|l|}{ Thumb chain } \\
\hline thumb_10 & 90 & 0 & 43.575 & 50 \\
\hline thumb_11 & $0 \leq \theta_{t 1} \leq 90$ & 0 & -0.34464 & -90 \\
\hline thumb_12 & $-180 \leq \theta_{t 2} \leq-90$ & 47 & 30 & 0 \\
\hline thumb_13 & $\theta_{t 2}+90$ & - & 0 & - \\
\hline
\end{tabular}
the same as the torque constant $K_{T}$.
Table 1. Modified Denavit-Hartenberg parameters.

Table 2. Nominal data of the gear-head D.C. Motor.

\begin{tabular}{lcc}
\hline \hline Characteristic & Symbol & Value \\
\hline Nominal voltage & $V_{N}$ & $12 \mathrm{~V}$ \\
Velocity without load & $\omega_{0}$ & $100 \mathrm{rpm}$ \\
Nominal torque & $\tau_{N}$ & $2 \mathrm{kgcm}$ \\
\hline \hline
\end{tabular}

Table 3. Measured parameters of the gear-head D.C. Motor.

\begin{tabular}{lcc}
\hline \hline Characteristic & Symbol & Value \\
\hline Armature resistance & $R_{a}$ & $12 \Omega$ \\
Current without load & $i_{0}$ & $39.5 \mathrm{~mA}$ \\
Dead zone & $V_{d z}$ & $0.46 \mathrm{~V}$ \\
Diameter of the motor pulley & $d_{m}$ & $7.5 \mathrm{~mm}$ \\
Diameter of the joint pulley & $d_{j}$ & $14 \mathrm{~mm}$ \\
\hline \hline
\end{tabular}

Table 4. Computed parameters of the gearhead D.C. Motor.

\begin{tabular}{lcc}
\hline \hline Characteristic & Symbol & Value \\
\hline Reduction & $n$ & 0.53574 \\
Maximum joint velocity & $\omega_{j_{\max }}$ & $5.609987 \mathrm{rad} / \mathrm{s}$ \\
Nominal torque on joints & $\tau_{j N}$ & $0.36624 \mathrm{Nm}$ \\
Armature constant & $K_{a}$ & $1.098766 \mathrm{Vs} / \mathrm{rad}$ \\
Torque Constant & $K_{T}$ & $1.098766 \mathrm{Nm} / \mathrm{A}$ \\
Maximum torque on joints & $\tau_{j_{\max }}$ & $1.968989 \mathrm{Nm}$ \\
Static friction & $f_{s}$ & $0.075478 \mathrm{Nm}$ \\
Viscous friction & $f$ & $0.336538 \mathrm{Nms} / \mathrm{rad}$ \\
\hline \hline
\end{tabular}


The maximum joint velocity is modeled in the URDF description as the velocity limit of the joint, the maximum torque on joints is modeled as the limit effort, the static friction is modeled as dynamics friction and the Viscous friction is modeled as dynamics damping.

While developing the dynamic model of a robot it is usual to consider the joint torques as inputs, neglecting the fact that, as a rule, D.C. motors, which inputs are the armature voltages, are used. Hence, it is supposed that the joint actuators are such that their torques can be directly commanded. The motivation for this is that, for the size of D.C motors typically used in robots, the armature inductance is small enough to be neglected, which also means that the electrical time constant is much faster then the mechanical time constant. Then, by neglecting the armature inductance, it is possible to model the D.C. motor as an ideal torque actuator subject to a viscous friction.

For permanent magnet D.C. motors, the generated torque is given by:

$$
\tau=K_{T} i_{a}
$$

where $K_{T}$ is the torque constant and $i_{a}$ is the armature constant.

The armature current is related with the voltage applied on the motor terminals by:

$$
v_{a}=R_{a} i_{a}+L_{a} \frac{d i_{a}}{d t}+e_{a}
$$

where $R_{a}$ is the armature resistance, $L_{a}$ is the armature inductance and $e_{a}$ is the back-electro-motion force, given by:

$$
e_{a}=K_{a} \dot{\theta}_{m}
$$

with $K_{a}$ the armature constant.

By replacing (7) in (6):

$$
v_{a}=R_{a} i_{a}+L_{a} \frac{d i_{a}}{d t}+K_{a} \dot{\theta}_{m}
$$

By taking the Laplace transform of (8) under null initial conditions and solving for $I_{a}(s)$ it results:

$$
I_{a}(s)=\frac{V_{a}(s)-s K_{a} \Theta_{m}(s)}{R_{a}+s L_{a}}
$$

and therefore, from (5):

$$
T(s)=K_{T} I_{a}(s)=K_{T}\left(\frac{V_{a}(s)-s K_{a} \Theta_{m}(s)}{R_{a}+s L_{a}}\right)
$$

which reflected to the joint side and neglecting the armature inductance is:

$$
\begin{aligned}
& T_{j}(s)=\frac{K_{T}}{n}\left(\frac{V_{a}(s)-s K_{a} \frac{\Theta_{l}(s)}{n}}{R_{a}}\right) \\
& =\frac{K_{T}}{n R_{a}} V_{a}(s)-s \frac{K_{T} K_{a}}{n^{2} R_{a}} \Theta_{l}(s)
\end{aligned}
$$

or, by returning to the time domain:

$$
\tau_{j}=\frac{K_{T}}{n R_{a}} v_{a}-\frac{K_{T} K_{a}}{n^{2} R_{a}} \dot{\theta}_{l}
$$

Obviously, each term on the right hand side of (12) has the dimension of torque. Hence, it is straightforward to conclude that the torque available on the joint is an equivalent torque produced by the actuator $\tau_{a}$, which is proportional to $v_{a}$ minus a viscous friction with coefficient $f_{a}$. That is:

$$
\tau_{j}=\tau_{a}-f_{a} \dot{\theta}_{l}
$$

with:

$$
\begin{aligned}
\tau_{a} & =\frac{K_{t}}{n R_{a}} v_{a} \\
f_{a} & =\frac{K_{T} K_{a}}{n^{2} R_{a}}
\end{aligned}
$$

The load torque on the joint side is given by the equivalent joint inertia, $J_{j}$ and the equivalent joint friction $f_{j}$. Hence,

$$
\tau_{j}=J_{j} \ddot{\theta}_{l}+f_{j} \dot{\theta}_{l}
$$

where $J_{j}=\frac{J_{m}}{n^{2}}+J_{l}$ is the equivalent inertia on the joint side, $f_{j}=\frac{f_{m}}{n^{2}}+f_{l}$ is the equivalent viscous friction on the joint side, $J_{m}$ and $f_{m}$ are the rotor inertia and viscous friction, respectively and $J_{l}$ and $f_{l}$ are the joint inertia and viscous friction, respectively.

Hence, from (13) and (16), the joint motion is described by:

$$
\tau_{a}=J_{j} \ddot{\theta}_{l}+\left(f_{j}+f_{a}\right) \dot{\theta}_{l}
$$

or, in the frequency domain:

$$
\frac{\Theta(s)}{T_{a}(s)}=\frac{1}{J_{j} s^{2}+s\left(f_{j}+f_{a}\right)}=\frac{1 / J_{j}}{s\left(s+\frac{f_{j}+f_{a}}{J_{j}}\right)}
$$

Whenever it is not possible to neglect the armature inductance, it is usual to implement a cascade controller, with a current control loop to command the actuator torque. Another possibility is to include the full actuator dynamics in the robot dynamic model as in Alves et al. (2018)

In the description of the joint in URDF, $\tau_{a}$ is the joint effort, hence its maximum value, computed with the maximum $v_{a}$ is the <limit effort> attribute. On the other hand, the minimum value of $\tau_{a}$, computed with the minimum value of $v_{a}$ is the dead-zone limit and can be represented by the <dynamics friction> attribute. The equivalent viscous friction on the joint side $f_{j}+f_{a}$ is represented in URDF by the <dynamics damping> attribute of the joint.

Note that the equivalent inertia on the joint side, $J_{j}$, is defined in the URDF description indirectly through the cumulative effect of the <inertia> and <origin> elements of the child link and the subsequent links in the kinematic chain.

\section{CONTROLLER DESIGN}

\subsection{Controllers in $R O S$}

The usual ROS structures such as nodes, messages, topics services and actions are designed for high level tasks and are not adequate for the implementation of controllers requiring real-time execution. Indeed, nodes do not execute in real-time and topics do not have any temporal restriction on the message delivery (Lages, 2016).

It is possible to overcome those limitations by using the ros_control ROS package (Chitta et al., 2017), which enables the implementation of controllers in a realtime safe approach. By using the ros_control package and the support of a real-time system such as 
RTAI (Bianchi et al., 1999), XENOMAI (Xenomai, 2012) or PREEMPT_RT (Open Software Automation Development Lab, 2012) it is possible to make the controllers to execute in real-time (Lages, 2016). The implementation of the controllers themselves are outside the scope of this work. Instead, this work is based on the use of the PID controllers implemented on the ros_controllers ROS package. See Lages (2016) for details on implementing controllers using other, possibly non-linear, control laws.

The controller manager handles the life-cycle of the controllers, and can load, unload, enable and disable controllers. It is important to note that the nomenclature of the ros_control package calls a controller any module that is loaded by the controller manager, which not necessarily implements the function of a controller in a control system (Barros and Lages, 2014). A typical example is the JointStateController. Its name indicates a state-space joint controller, but actually it is a sensor which publishes the position, velocity and torque on joints.

From the point of view of digital control theory, the ros_ control framework uses the paradigm of a continuous control law implemented in a digital computer with a sampling rate fast enough for neglecting the effects of discretization (Lages, 2016). Hence, the controller should be designed as a continuous time controller.

\subsection{Controller Tuning}

Here the PID controllers implemented in ROS are used. In section 2.2 hand joints are modeled as actuated in torque. Since the purpose here is to control the joint position, a controller called effort_controllers/ JointPositionController in ROS nomenclature is used. However, this controller is not installed in the default ROS Desktop installation. Hence, the ros_controllers package should be installed. This packages uses the PID library available in the control_toolbox package.

It is usual in ROS community to use the dynamic_ reconfigure package to tune the PID gains by experimentation. Even tutorials use this approach. Needless to say that this procedure is frustrating and leads to the perception that tuning PID controllers is a difficult task and other controllers are easier to tune. On the other hand, here the approach is to compute the equivalent joint inertia and viscous friction, find the transfer function of the joint and design the PID gains.

The joint viscous friction can be computed by (15). To compute the equivalent joint inertia, recall that all joints of each finger (with exception of the thumb) are actuated by a single actuator. Hence, the equivalent joint inertia is the result of reflecting the inertia of each link to the first joint of the finger. Furthermore, the mechanical design of index, middle, ring and little fingers are the same. The difference is just that each one of them is mounted in a different position and orientation on the hand. Therefore, the same PID gains can be used for them.

The center of mass of combined links 2 and 3 is given by: ${ }^{2} P_{c 23}=\left({ }^{2} P_{c 2} m_{2}+\left({ }^{2} R_{3}\left(\theta_{3}\right){ }^{3} P_{c 3}+{ }^{2} P_{3}\right) m_{3}\right) /\left(m_{2}+m_{3}\right)$

where ${ }^{i} P_{c j}$ is the position of center of mass of link $j$ with respect the link $i$ frame and $m_{i}$ is the mass of link $i$.
The position of the center of mass of link 3 with respect to the center of mass of combined links 2 an 3 is:

$$
{ }^{c 23} P_{c 3}=\left({ }^{2} R_{3}\left(\theta_{3}\right){ }^{3} P_{c 3}+{ }^{2} P_{3}\right)-{ }^{2} P_{c 23}
$$

By using the parallel axis theorem, the inertia tensor of link 3 at the center of mass of combined links 2 and 3 can be computed as:

$$
{ }^{c 23} I_{3}={ }^{c 3} I_{3}+m_{3}\left({ }^{c 23} P_{c 3}^{T}{ }^{c 23} P_{c 3} I-{ }^{c 23} P_{c 3}{ }^{c 23} P_{c 3}^{T}\right)
$$

Similarly, the position of the center of mass of link 2 with respect to the center of mass of combined links 2 an 3 is:

$$
{ }^{c 23} P_{c 2}={ }^{2} P_{c 2}-{ }^{2} P_{c 23}
$$

and again, by using the parallel axis theorem, the inertia tensor of link 2 at the center of mass of combined links 2 and 3 can be computed as:

$$
{ }^{c 23} I_{2}={ }^{c 2} I_{2}+m_{2}\left({ }^{c 23} P_{c 2}^{T}{ }^{c 23} P_{c 2} I-{ }^{c 23} P_{c 2}{ }^{c 23} P_{c 2}^{T}\right)
$$

Then, the inertia tensor of combined links 2 and 3 is:

$$
{ }^{c 23} I_{23}={ }^{c 23} I_{3}+{ }^{c 23} I_{2}
$$

The same procedure can be used to reflect $m_{2}+m_{3},{ }^{2} P_{c 23}$ and ${ }^{c 23} I_{23}$ to joint 1 and combine them with $m_{1},{ }^{1} P_{c 1}$ and ${ }^{c 1} I_{c 1}$ to compute $m_{123}=m_{1}+m_{2}+m_{3},{ }^{1} P_{c 123}$ and ${ }^{c 123} I_{123}$.

Finally, by using the parallel axis theorem again, the total combined inertia can be reflected to the joint 1 :

$$
{ }^{1} I_{123}={ }^{c 123} I_{123}+m_{123}\left({ }^{1} P_{c 123}^{T}{ }^{1} P_{c 123} I-{ }^{1} P_{c 123}{ }^{1} P_{c 123}^{T}\right)
$$

Then, as the joint 1 axis is the $\mathrm{Z}$ axis, the equivalent inertia is given by the element $i_{33}$ of ${ }^{1} I_{123}$ and from (18), the transfer function of the joint is:

$$
G(s)=\frac{1 / i_{33}}{s\left(s+f_{a} / i_{33}\right)}
$$

The joint parameters, transfer function and PID gains, computed by using the Matlab pidtune function, with a crossover frequency of $100 \mathrm{rad} / \mathrm{s}$, are shown in table 5.

Table 5. Joint parameters, transfer function and PID gains.

\begin{tabular}{lccc}
\hline \hline Parameter $\backslash$ Joint & thumb1 & thumb2 & $\begin{array}{c}\text { index, middle } \\
\text { ring and little }\end{array}$ \\
\hline Friction $\left(\mathrm{kgm}^{2} / \mathrm{s}\right)$ & 0.336538 & 0.336538 & 0.336538 \\
Inertia $\left(\mathrm{kgm}^{2}\right)$ & 0.9731 & 0.2342 & 0.5490 \\
Transfer function & $\frac{10280}{s(s+3458)}$ & $\frac{42690}{s(s+14370)}$ & $\frac{18210}{s(s+6131)}$ \\
P gain & 29.6 & 29.3 & 29.4 \\
I gain & 1600 & 1660 & 1640 \\
D gain & 0 & 0 & 0 \\
\hline \hline
\end{tabular}

\section{SIMULATION}

The hand was simulated in Gazebo (Koenig and Howard, 2004), by using the PID controllers implemented in ROS. Gazebo does not natively support mimic joints and the gazebo_ros_control plugin, used to interface Gazebo to ROS, does not support them either. Therefore, MimicJointPlugin from the Robotics Group of the University of Patras <https: //github.com/roboticsgroup/ roboticsgroup_gazebo_plugins $>$ is used. 
It is important to note that the default integration step size used by the Gazebo simulator is $1 \mathrm{~ms}$. However, due to the small inertia moments of the fingers, the fastest pole of the joint transfer functions is at $14370 \mathrm{rad} / \mathrm{s}$ or $2.2871 \mathrm{kHz}$, which means a period of $437.2 \mu \mathrm{s}$. Therefore, the Gazebo default integration step size is too large and makes the simulation to diverge. Gazebo signalizes this by exploding the robot. Hence, the Gazebo integration step size was reduced to $30 \mu$ s to correct the problem.

Figure 4 shows the hand model in Gazebo. The hand is in open position. The joint values are $0 \mathrm{rad}$ for the thumb1 joint and $-\pi / 2$ rad for the thumb2, index, middle, ring and little joints. The joint range values are described in table 1 .

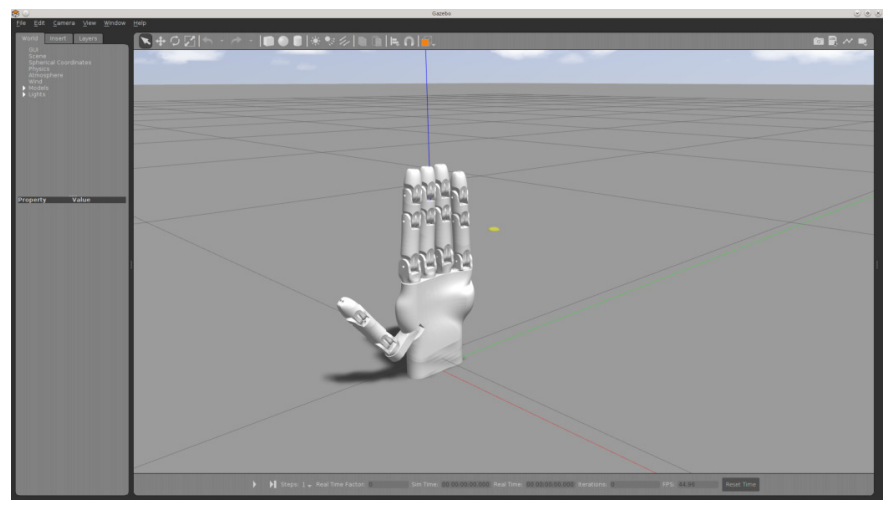

Figure 4. The Miitzhand in Gazebo.

Figure 5(a) shows the hand with the index finger at $-3 \mathrm{rad}$. Then, a step of $1 \mathrm{rad}$ is applied to the reference of the index joint controller by publishing a value of $-2 \mathrm{rad}$ to the /index_controller/command topic. The finger moves to the position shown in Figure 5(b). The index joint position and torque (note that all joints move together for the index, middle, ring and little finger) can be obtained through the /joint_positions topic or though the /index_controller/status topic.

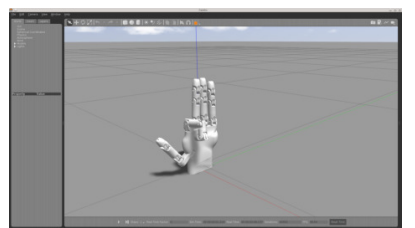

(a) Index finger at $-3 \mathrm{rad}$.

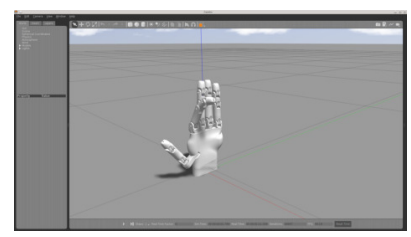

(b) Index finger at $-2 \mathrm{rad}$.
Figure 5. Motion of $1 \mathrm{rad}$ in the index finger.

Figure 6 shows plot of the joint reference and the position of index finger, while executing the motion. Figure 7 shows the torque commanded by the controller. The curves do not appear to be the typical second order response. Indeed, what is happening is that $1 \mathrm{rad}$ is too large for a step. Gazebo simulates the torque saturation at $1.968989 \mathrm{Nm}$ (see Table 4), thus it imposes a constant velocity until the commanded torque returns to the limits. Note that the /joint_positions topic reports the effective joint torque used for simulation, while the /index controller/status reports the torque commanded by the controller.

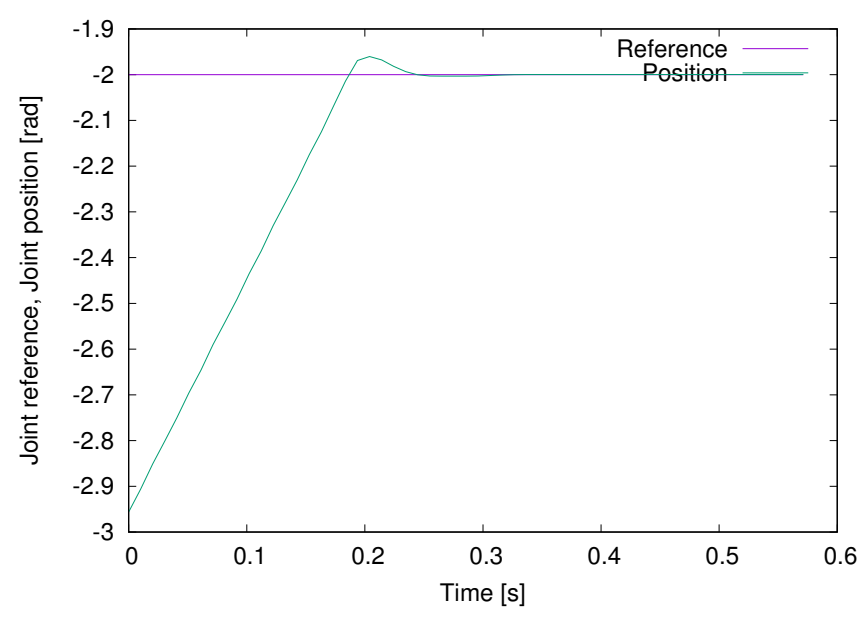

Figure 6. Joint reference and position for the index finger.

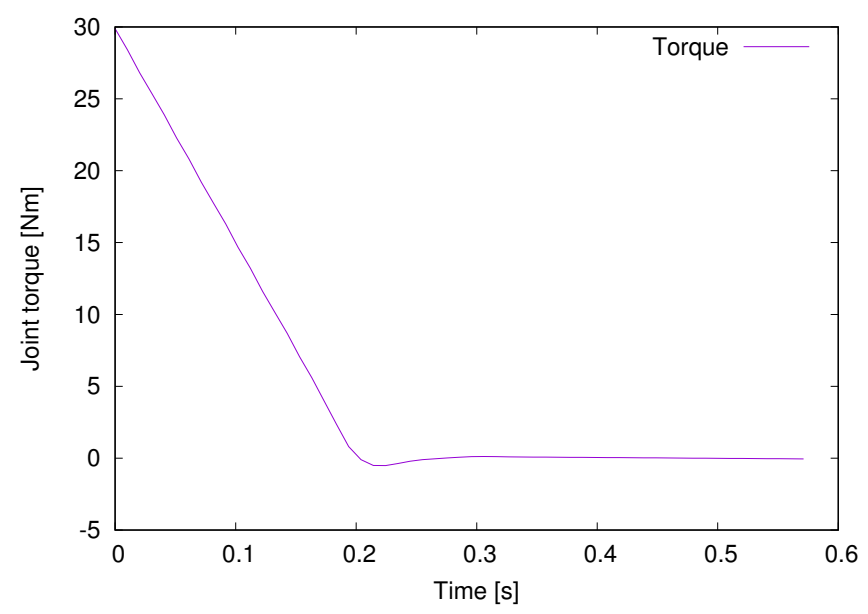

Figure 7. Joint torque for the index finger.

Figures 8 and 9 show the same plots for a smaller step of $0.1 \mathrm{rad}$. Now, the torque is under the maximum limits and no saturation is imposed by Gazebo. Hence, a typical second order system response can be observed.

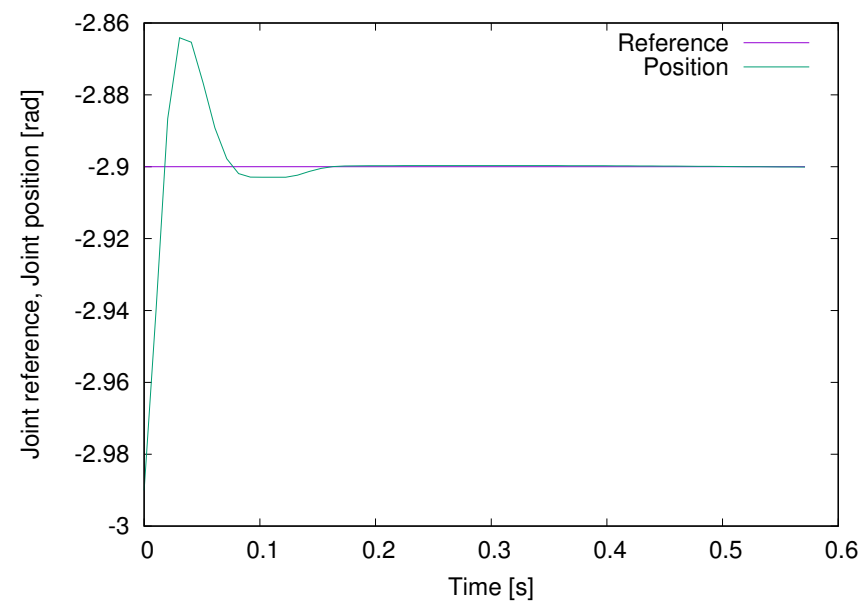

Figure 8. Joint reference and position for index finger for a small step. 


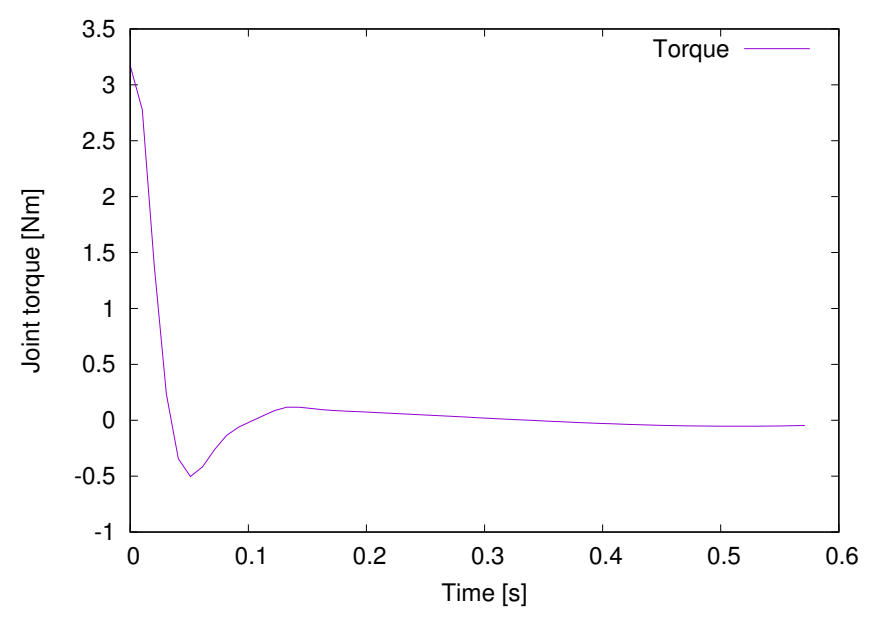

Figure 9. Joint torque for index finger for a small step.

\section{CONCLUSIONS}

This work presented the development of a robotic hand, including modeling in ROS, visualization in RViz, controller design and simulation in Gazebo.

A PID controller was used to control the hand joints. The controller gains were tuned for the desired performance and results showed that it was able to track the reference with null steady state error.

For future works some directions are to test the hand in performing actual prehensions, to define the affordances related to each prehension type and to implement more advanced controllers, such as computed torque or impedance controllers.

\section{REFERENCES}

Alves, T.G., Lages, W.F., and Henriques, R.V.B. (2018). Non-linear pose stabilization controller for a differntialdrive mobile robot: Optimization-based controller tuning. In Proceedings of $12^{\text {th }}$ International IFAC Symposium on Robot Control. International Federation of Automatic Control, Budapest, Hungary.

An, K., Chao, E., Cooney, W., and Linscheid, R. (1979). Normative model of human hand for biomechanical analisys. Journal of biomechanics, 12, 775-788.

Barros, T.T.T. and Lages, W.F. (2014). A mobile manipulator controller implemented in the robot operating system. In Proceedings for the Joint Conference of $45^{\text {th }}$ International Symposium on Robotics and $8^{\text {th }}$ German Conference on Robotics, 121-128. VDE Verlag, Munich, Germany. ISBN 978-3-8007-3601-0.

Becker, J.C., Thakor, N.V., and Gruben, K.V. (1986). A study of humand hand tendom kinematics with applications to robot hand design. IEEE, 1540-1545.

Bianchi, E., Dozio, L., Ghiringhelli, G.L., and Mantegazza, P. (1999). Complex control systems, applications of DIAPM-RTAI at DIAPM. In Proceedings of the Realtime Linux Workshop. Vienna. <http://www.rtai. org>.

Brook, N., Mizrahi, J., Shoham, M., and Dayan, J. (1995). A biomechanical model of index finger dynamics. Med. Eng. Phys, 17, 54-63.
Bucholz, B. and Armstrong, T. (1992). A kinematic model of the human hand torso evaluate its prehensile capabilities. Biomechanics Journal, 25, 149-162.

Chitta, S., Marder-Eppstein, E., Meeussen, W., Pradeep, V., Rodríguez Tsouroukdissian, A., Bohren, J., Coleman, D., Magyar, B., Raiola, G., Lüdtke, M., and Perdomo, E.F. (2017). ros_control: A generic and simple control framework for ros. The Journal of Open Source Software. doi:10.21105/joss. 00456. URL <http://www.theoj.org/joss-papers/ joss.00456/10.21105. joss.00456.pdf $>$.

Cousins, S. (2010). Welcome to ros topics. IEEE Robotics and Automation Magazine, 17(1), 13-14.

Craig, J.J. (1989). Introduction to Robotics Mechanics and Control. Addison-Wesley, 2nd edition.

Cutkosky, M. (1989). On grasp choice, grasp models, and the design of hands for manufacturing tasks. Robotics and Automation, IEEE Transactions, 5, 269-279.

Fu, K.S., Gonzales, R.C., and Lee, C.S.G. (1987). Robotics Control, Sensing, Vision and Intelligence. Industrial Engineering Series. McGraw-Hill, New York.

Hasser, C.J. and Cutkosky, M.R. (2002). System identification of the human hand grasping a haptic knob. In Proceedings of the 10th Symposium on Haptic Interfaces for Virtual Environment and Teleoperator Systems (HAPTICS 2002).

Kapandji, I.A. (2009). The Physiology of the Joints: Upper Limb, volume 1. Churchill Livingstone, Edinburgh, 6th edition.

Koenig, N. and Howard, A. (2004). Design and use paradigms for gazebo, an open-source multi-robot simulator. In Proceedings of the 2004 IEEE/RSJ International Conference on Intelligent Robots and Systems (IROS 2004), volume 3, 2149-2154. IEEE Press, Sendai, Japan. doi:10.1109/IROS.2004.1389727.

Lages, W.F. (2016). Implementation of real-time joint controllers. In A. Koubaa (ed.), Robot Operating System (ROS): The Complete Reference (Volume 1), volume 625 of Studies in Computational Intelligence, 671-702. Springer International Publishing, Switzerland.

Magee, D. (1997). Orthopedic Physical Assessment. W. B. Saunders, 3th edition edition.

Napier, J.R. (1980). Hands. George Allen and Unwin, London, England.

Open Software Automation Development Lab (2012). Osadl project: Realtime linux. <https://www.osadl. org/Realtime-Linux.projects-realtime-linux.0. html>.

ROS.org (2013). SolidWorks URDF exporter. Available at: <http://wiki.ros.org/sw_urdf_exporter>. Access: December 2013.

Taylor, G.L. and Schwartz, R.J. (1955). The anatomy and mechanics of the human hand. Artificial Limbs, 2, 2235.

Xenomai (2012). Xenomai: Real-time framework for linux. <http://www xenomai.org>. 